\title{
Incidence of intracerebral and subarachnoid haemorrhage in southern Sweden
}

\author{
O G Nilsson, A Lindgren, N Ståhl, L Brandt, H Säveland
}

\begin{abstract}
Objectives-Spontaneuos intracranial haemorrhage - that is, mainly subarachnoid haemorrhage (SAH) and primary intracerebral haemorrhage (PICH)constitutes an important part of all strokes. As previous epidemiological studies have demonstrated highly variable incidence rates, we conducted a large prospective investigation of all haemorrhagic strokes during a 1 year period.

Methods-Twelve hospitals serving a defined population of 1.14 million in southern Sweden registered all cases with spontaneous intracranial haemorrhage, including those found dead outside hospitals, during 1996. All patients were examined with CT of the brain or underwent necropsy. Incidence rates adjusted to the Swedish population for age and sex, as well as location of haematoma and prevalence of risk factors were calculated.

Results-A total of 106 patients with SAH and 341 patients with PICH were identified. The annual incidence $/ 100000$ was 10.0 (6.4 for men and $\mathbf{1 3 . 5}$ for women) for SAH and 28.4 (32.2 for men and 24.7 for women) for PICH when adjusted to the Swedish population. Subarachnoid haemorrhage affected twice as many women as men. The incidence of both types of haemorrhage increased with advancing age, but in particular, this was the case for supratentorial PICH. Lobar haematomas were the most common (51.6\%) type of PICH. Among patients with PICH, 37\% had hypertension, $41 \%$ other vascular disease, and $12 \%$ were on oral anticoagulation. Among patients with SAH, $28 \%$ had hypertension and $18 \%$ vascular disease before the haemorrhage but no one was on treatment with oral anticoagulation.

Conclusions-The incidence of PICH was high, especially for the older age groups. PICH was, on average, three times as common as SAH. The study underscores the importance of PICH and SAH as significant stroke subgroups.

(F Neurol Neurosurg Psychiatry 2000;69:601-607)
\end{abstract}

Keywords: incidence; intracerebral haemorrhage; subarachnoid haemorrhage

Spontaneous intracranial haemorrhage constitutes an important part of stroke because of its high morbidity and mortality. Although primary intracerebral haemorrhage (PICH) together with subarachnoid haemorrhage $(\mathrm{SAH})$ represent only $10 \%-15 \%$ of all strokes, they have been estimated to cause more than $50 \%$ of the overall stroke mortality. ${ }^{1}$

The natural history and management of $\mathrm{SAH}$ is relatively well established and documented but less is known about PICH. ${ }^{2}$ The introduction of brain imaging using CT dramatically improved stroke diagnosis and subsequent incidence studies resulted in a major reassessment of PICH. It has been suggested that previous studies based on clinical criteria alone underestimated the frequency of small haematomas, which were interpreted as ischaemic infarcts, and large infarcts were often diagnosed as intracerebral haematomas. $^{3-5}$ Despite a high utilisation rate of CT $(>80 \%)$ in patients with acute stroke in more recent studies, the reported incidence rates for PICH may vary up to five times between studies (from nine to 43 cases/100 000 population/year).$^{6-12}$ Part of the variability has been attributed to population differences in age or race distribution. ${ }^{10}{ }^{13}$ However, most of these studies have been based on few cases (usually<100). Different methods for sampling data, including retrospective analysis as well as prospective hospital and population based designs, may also cause varying results. Highly variable incidence rates have also been reported for SAH; from about 6 to over 30/100 000/year. ${ }^{8}{ }^{14-17}$ Because of the substantial medical, socioeconomic, and personal consequences of PICH and SAH, there is a need for further large prospective population based studies.

We prospectively registered all cases of spontaneous intracranial haemorrhage-that is, SAH, PICH, bleeding in tumour, or haemorrhage caused by an arteriovenous malformation (AVM), during one calendar year (1996) in a defined population in southern Sweden. Incidence, prevalence of risk factors, and localisation of haematoma are presented.

\section{Patients and methods}

Twelve hospitals, including four forensic/ pathology departments serving a defined population of 1.14 million in southern Sweden participated from 1 January to 31 December 1996. Before the recruitment of patients started, all participating hospitals were contacted and agreed to participate in the study. For each case with a stroke, either admitted to or occurring at a hospital, CT was perfomed within 1-2 days. If CT (or necropsy) disclosed an intracranial haemorrhage, special study forms were filled out immediately during the acute phase by a qualified physician (from a department of neurology, internal medicine, pathology, or our own department of neurosurgery) and sent to 
the authors for further analysis. All patients diagnosed with spontaneous intracranial haemorrhage - that is, $\mathrm{PICH}, \mathrm{SAH}$, bleeding in tumour, or bleeding caused by an AVM - were registered. Computed tomography is routinely used for the evaluation of acute stroke at all hospitals participating in the study. Paediatric cases with intracranial haemorrhage were detected via paediatric neurosurgeons at our department. Fatal cases in the community were searched for at the forensic/pathology departments covering the study population. Thus all patients included in the study had been investigated by either CT or necropsy. Angiography was carried out in patients with SAH, and in those with intracerebral haemorrhage in whom age, haematoma site, or clinical situation was suggestive of an AVM or aneurysm as the cause.

Brain CT were evaluated by one of us (OGN) or by a radiologist. Primary intracerebral haemorrhage was defined as a focal collection of blood in the brain parenchyma seen as a uniform high attenuation area on CT unrelated to tumour or trauma, or, alternatively, an adequate necropsy examination showing an intracerebral haemorrhage. The location of PICH was categorised as lobar (cortical or subcortical), central (caudate, putamen, globus pallidus, thalamus, internal capsule, deep periventricular white matter, or intraventricular), cerebellar, or of brainstem origin. Intracerebral haematomas caused by a ruptured aneurysm were allocated to the SAH group and those caused by an arteriovenous malformation (AVM) were also categorised separately. Subarachnoid haemorrhage was defined as blood in the subarachnoid space shown on CT or at necropsy, or a clinical history and examination consistent with $\mathrm{SAH}$ together with typical findings in the CSF (xantochromia and many red blood cells). Haemorrhage related to a brain tumour was categorised separately. Suspicion of a traumatic cause of the haemorrhage excluded the patient from the study, thereby excluding cases with subdural haematomas. Patients with haemorrhagic conversion of a cerebral infarct were not recorded.

The following possible risk factors obtained from the anamnestic information were recorded: (1) arterial hypertension when the patient was on antihypertensive medication at the time of bleeding or had previously repeated measurements of blood pressure $>160 / 95 \mathrm{~mm}$ $\mathrm{Hg}$; (2) diabetes mellitus, including both insu- lin dependent and non-insulin dependent; (3) previous cerebrovascular disease, ischaemic or haemorrhagic; (4) previously known heart disease, including coronary disease and atrial fibrillation; (5) peripheral arteriopathy-that is, ischaemic leg pain or ulcers; (6) ongoing treatment with oral anticoagulant (warfarin, dicumarol) or antiplatelet (acetylsalicylic acid) drugs.

Incidence rates were sex and age adjusted to the Swedish population of 1996 using the direct method with demographic data obtained from the local health authority. Incidence rates were also age adjusted to the European and world populations. ${ }^{18}$ 95\% Confidence intervals (95\% CIs) were calculated from the Poisson distribution. Poisson regression was performed for comparison of incidence rates. ${ }^{19}$ Group comparisons of risk factors were performed using $\chi^{2}$ or Kruskal-Wallis tests.

\section{Results}

OVERALL INCIDENCE

A total of 466 patients with spontaneous intracranial haemorrhage were diagnosed during 1996. There were 341 cases $(73.2 \%)$ of PICH, 106 cases $(22.7 \%)$ of SAH, six cases (1.3\%) of haemorrhage caused by AVM (diagnosed by angiography), and 13 cases $(2.8 \%)$ of bleeding in brain tumour.

\section{INCIDENCE OF SAH}

Among the 106 patients with SAH, 81 (76\%) were caused by rupture of an intracranial aneurysm as demonstrated angiographically or at necropsy. In this report, we do not distinguish between aneurysmal and nonaneurysmal SAH. The age and sex specific incidence rates with $95 \%$ CIs are shown in table 1 and fig 1 . Overall annual incidence rate was 9.3/100 000 in southern Sweden and 10.0/ 100000 when adjusted to the Swedish population. Median age was 60 years. Sex specific annual incidence rates were 12.3/100 000 for women and 6.2/100 000 for men, and when adjusted to the Swedish population 13.5/100 000 and 6.4/100 000, respectively. Thus, 71 $(67 \%)$ of the patients with SAH were women and there was a significant difference in incidence rate between men and women (incidence rate ratio from Poisson regression: 2.00, 95\% CI 1.31-3.07, $\mathrm{p}=0.006)$. For both sexes, the main increase in incidence rate occurred from young adulthood to middle age (in the

Table 1 Age and sex specific incidence rates (/100000/y) for subarachnoid haemorrhage in southern Sweden 1996

\begin{tabular}{|c|c|c|c|c|c|c|c|c|c|}
\hline \multirow[b]{2}{*}{ Age } & \multicolumn{3}{|c|}{ Male } & \multicolumn{3}{|c|}{ Female } & \multicolumn{3}{|c|}{ Total } \\
\hline & $n$ & Rate & $95 \% C I$ & $n$ & Rate & $95 \% C I$ & $n$ & Rate & $95 \% C I$ \\
\hline $0-14$ & 0 & 0 & & 0 & 0 & & 0 & 0 & \\
\hline $15-24$ & 0 & 0 & & 1 & 1.4 & $0.0-8.0$ & 1 & 0.7 & $0.0-3.9$ \\
\hline $25-34$ & 0 & 0 & & 2 & 2.7 & $0.3-9.8$ & 2 & 1.3 & $0.2-4.7$ \\
\hline $35-44$ & 3 & 4.1 & $0.8-11.9$ & 6 & 8.4 & $3.1-18.2$ & 9 & 6.2 & $2.8-11.7$ \\
\hline $45-54$ & 11 & 13.2 & $6.6-23.6$ & 18 & 22.2 & $13.2-35.1$ & 29 & 17.6 & $11.8-25.3$ \\
\hline $55-64$ & 8 & 13.8 & $6.0-27.2$ & 13 & 22.2 & $11.8-37.9$ & 21 & 18.0 & $11.1-25.3$ \\
\hline $65-74$ & 8 & 16.5 & $7.1-32.5$ & 14 & 25.3 & $13.8-42.5$ & 22 & 21.2 & $13.3-32.1$ \\
\hline $75-84$ & 4 & 12.8 & $3.5-32.8$ & 12 & 27.1 & $14.0-47.4$ & 16 & 21.2 & $12.1-34.4$ \\
\hline $85-$ & 1 & 12.3 & $0.3-68.6$ & 5 & 27.8 & $9.0-65.0$ & 6 & 23.0 & $8.4-50.1$ \\
\hline Total & 35 & $6.2 / 6.4$ & $4.3-8.6$ & 71 & $12.3 / 13.5$ & $9.6-15.6$ & 106 & $9.3 / 10.0$ & $7.6-11.2$ \\
\hline
\end{tabular}

Values in italics are adjusted to the Swedish population. $\mathrm{n}$ Indicates number of cases. $95 \% \mathrm{CI}=95 \%$ confidence interval (Poisson distribution). 


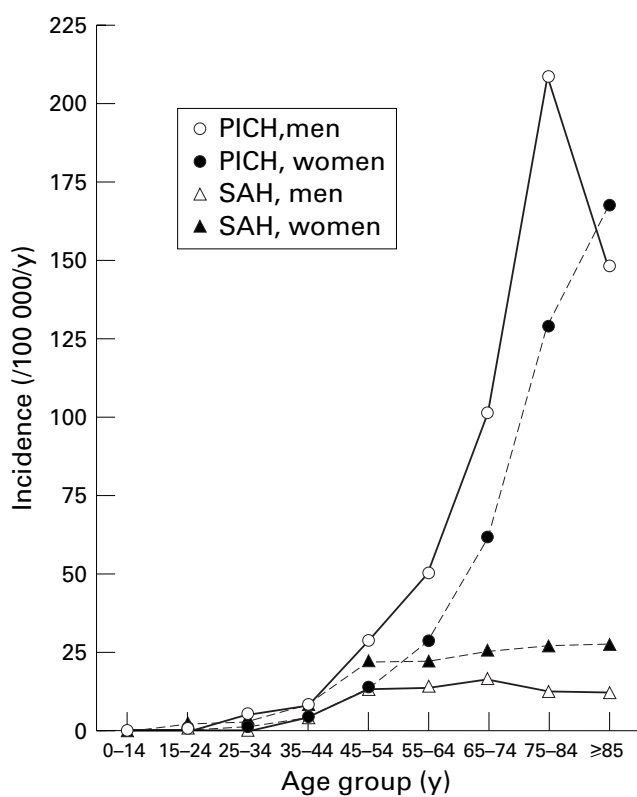

Figure 1 Age and sex specific incidence rates 100 000/year for primary intracerebral haemorrhage (PICH) and subarachnoid haemorrhage (SAH).

35-44 and 45-54 age groups), after which it remained largely constant (fig 1). Incidence rates for the older age groups ( $>55$ years) were $22.2-27.8 / 100000 /$ year for women and 12.3$16.5 / 100000 /$ year for men. Age adjustment to the European and world populations resulted in incidence rates of 7.9 and 5.9/100 000/year, respectively (both sexes combined).

Thirteen $(12 \%)$ of the patients with SAH had, in addition, an intraparenchymatous haematoma caused by the ruptured aneurysm.

INCIDENCE OF PICH

Age and sex specific incidence rates for PICH (excluding haemorrhages caused by aneurysm, AVM, or tumour) are presented in table 2 and fig 1 . The overall incidence for PICH/100 000/ year was 33.5 for men, 26.6 for women, and 30.0 for both sexes combined. When adjusted to the Swedish population the rates were 32.2 (men), 24.7 (women), and 28.4 (men and women)/100 000/year. Women were significantly less likely to have a PICH than men (incidence rate ratio from Poisson regression: $0.79,95 \%$ CI $0.63-0.99, \mathrm{p}=0.029$ ) As illustrated in fig 1 , there was a very pronounced relation with advancing age for both sexes with annual incidence rates of up to 208.1/100 000

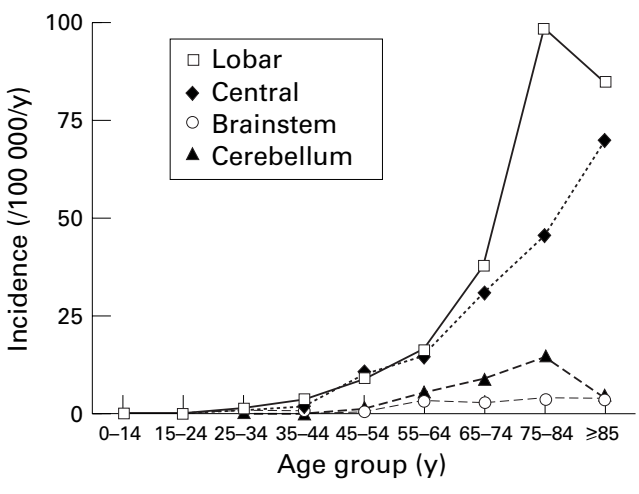

Figure 2 Age specific incidence rates/100 000/year of primary intracerebral haemorrhage (PICH) by location.

men in the $75-84$ year group and $167.0 / 100$ 000 women in the $\geqslant 85$ group. There seemed to be lower incidence rates for men in the highest age group $(\geqslant 85)$ compared with the $75-84$ year old group, but this was not statistically significant (Poisson regression). When adjusted for age to the European and world populations, overall incidence rates were 20.6 and 13.9/100 000/year, respectively.

LOCATION OF HAEMATOMA

Lobar haematomas were diagnosed in $51.6 \%$ $(\mathrm{n}=176)$ of patients with PICH $(\mathrm{n}=341)$. Central haematomas constituted $35.5 \%(n=121)$, whereas only $4.4 \%(n=15)$ occurred in the brainstem and $8.5 \%(n=29)$ in the cerebellum. As seen in fig 2, there was a strong influence of age on the incidence of particularly lobar and central haematomas. However, statistical analysis using Poisson regression testing changes in incidence rate for different age groups and haematoma locations could not be performed due to the low number of brain stem haematomas. Nevertheless, lobar haematomas accounted for $42 \%(34 / 81)$ of all PICH in the 45-54 and 55-64 year groups combined, whereas they constituted $52 \%(96 / 163)$ of PICH among those older than 75. Moreover, lobar haematomas were 2.6 times as common as brain stem and cerebellar haematomas (combined) in the 45-54 and 55-64 year groups, but six times as common among those older than 75 . There was no statistically significant impact of sex on location of haematoma $\left(\chi^{2}, \mathrm{p}=0.34\right)$.

Table 2 Age and sex specific incidence rates (/100000/y) for primary intracerebral haemorrhage in southern Sweden 1996

\begin{tabular}{|c|c|c|c|c|c|c|c|c|c|}
\hline \multirow[b]{2}{*}{ Age } & \multicolumn{3}{|c|}{ Male } & \multicolumn{3}{|c|}{ Female } & \multicolumn{3}{|c|}{ Total } \\
\hline & $n$ & Rate & $95 \% C I$ & $n$ & Rate & $95 \% C I$ & $n$ & Rate & $95 \% C I$ \\
\hline $0-24$ & 0 & 0 & & 0 & 0 & & 0 & 0 & \\
\hline $25-34$ & 4 & 5.1 & $1.4-13.0$ & 1 & 1.4 & $0.0-7.6$ & 5 & 3.3 & $1.1-7.6$ \\
\hline $35-44$ & 6 & 8.2 & $3.0-17.7$ & 3 & 4.2 & $0.9-12.2$ & 9 & 6.2 & $2.8-11.7$ \\
\hline $45-54$ & 24 & 28.8 & $18.4-42.8$ & 11 & 13.6 & $6.8-24.3$ & 35 & 21.3 & $14.8-29.6$ \\
\hline $55-64$ & 29 & 50.0 & $33.5-71.8$ & 17 & 29.0 & $16.9-46.5$ & 46 & 39.4 & $28.9-52.6$ \\
\hline $65-74$ & 49 & 101.1 & $74.8-133.6$ & 34 & 61.5 & $42.6-85.9$ & 83 & 80.0 & $63.7-99.2$ \\
\hline $75-84$ & 64 & 208.1 & $160.6-265.2$ & 57 & 128.9 & $97.6-167.1$ & 121 & 161.7 & $134.3-193.1$ \\
\hline $85-$ & 12 & 147.8 & $69.7-237.3$ & 30 & 167.0 & $112.7-238.4$ & 42 & 161.0 & $116.0-217.8$ \\
\hline Total & 188 & $33.5 / 32.2$ & $28.9-38.6$ & 153 & $26.6 / 24.7$ & $22.6-31.2$ & 341 & $30.0 / 28.4$ & $26.9-33.3$ \\
\hline
\end{tabular}

Values in italics are adjusted to the Swedish population. $\mathrm{n}$ Indicates number of cases. $95 \% \mathrm{CI}=95 \%$ Confidence interval (Poisson distribution). 
Table 3 Prevalence of possible risk factors for primary intracerebral haemorrhage and subarachnoid haemorrhage in southern Sweden 1996

\begin{tabular}{|c|c|c|c|c|c|c|}
\hline \multirow[b]{2}{*}{ Risk factor } & \multicolumn{5}{|c|}{ Primary intracerebral haemorrhage } & \multirow{2}{*}{$\begin{array}{l}\text { Subarachnoid } \\
\text { haemorrhage }\end{array}$} \\
\hline & Lobar & Central & Brainstem & Cerebellum & Total & \\
\hline Age (median, y) & 75 & 72 & 62 & 72 & 74 & 60 \\
\hline Sex ( $\%$ female $)$ & 48.9 & 40.5 & 46.7 & 34.5 & 44.6 & 67.0 \\
\hline Hypertension & 34.1 & 37.8 & 60.0 & 39.3 & 37.0 & 28.3 \\
\hline Diabetes mellitus & 9.1 & 11.8 & 13.3 & 3.6 & 9.8 & 3.8 \\
\hline Vascular disease (total) & 45.1 & 36.4 & 40.0 & 37.0 & 41.1 & 17.9 \\
\hline Prior stroke & 19.4 & 18.2 & 26.7 & 11.1 & 18.6 & 6.6 \\
\hline Heart disease & 30.3 & 25.6 & 26.7 & 22.2 & 27.8 & 11.3 \\
\hline Peripheral arteriopathy & 3.4 & 0.8 & 0 & 7.4 & 2.1 & 0 \\
\hline Anticoagulation & 15.4 & 7.6 & 6.7 & 14.3 & 11.9 & 0 \\
\hline Antiplatelet therapy & 22.3 & 24.2 & 20 & 10.7 & 21.9 & 4.7 \\
\hline Number of patients (\%) & $176(51.6)$ & $121(35.5)$ & $15(4.4)$ & $29(8.5)$ & 341 & 106 \\
\hline
\end{tabular}

Values are percentages (except for age and number of patients).

RISK FACTORS

The prevalence of various risk factors is presented in table 3 for the different locations of PICH and SAH. The median age for the 106 patients with SAH was 60 years and $67 \%$ $(n=71)$ were women. For SAH, a history of arterial hypertension was documented in 30 cases $(28 \%)$, previous vascular disease in 19 cases $(18 \%$; prior stroke seven and heart disease 12 cases) and diabetes mellitus in four cases $(4 \%)$. Five per cent $(n=5)$ of patients with $\mathrm{SAH}$ were on antiplatelet (acetylsalicylic acid) therapy whereas no one was treated with anticoagulation.

Among the different haematoma locations for patients with PICH, there was no significant difference in age (Kruskal-Wallis test, $\mathrm{p}>0.05)$ or prevalence of the various risk factors $\left(\chi^{2}\right.$ tests, $\left.p>0.05\right)$. The results for risk factors are, therefore, described for the whole material from patients with $\mathrm{PICH}$, although data for each haematoma subtype are presented in table 3. The 341 patients with PICH had a median age of 74 years, among which $153(45 \%)$ were women. Hypertension had been documented before the bleeding in 126 cases $(37 \%)$, vascular disease in 140 cases $(41 \%)$ (prior stroke 63 cases $(19 \%)$, heart disease 95 cases $(28 \%)$, peripheral arteriopathy seven cases $(2 \%))$, and diabetes mellitus in 33 cases $(10 \%)$. Antiplatelet therapy was found in $75(22 \%)$ of the patients with PICH, and 41 $(12 \%)$ were treated with anticoagulant drugs.

STATUS AT ADMISSION

Among the 106 patients with SAH (including non-aneurysmal cases), 66 (62\%) were alert or only slightly drowsy or confused, $10(9 \%)$ were very drowsy, and 26 (25\%) were unconscious when arriving at the hospital. The remaining cases $(n=4 ; 4 \%)$ died outside hospital.

Of the 341 patients with PICH, 223 (65\%) were alert or only slightly drowsy or confused at admission to hospital. Twenty five $(7 \%)$ were very drowsy and $64(19 \%)$ were unconscious (information on level of consciousness was not obtained in eight cases). Twenty one patients (6\%) with PICH died outside hospital.

\section{Discussion}

The present study is probably one of the largest prospective investigations on the incidence of $\mathrm{PICH}$ and SAH, with a study population of 1.14 million. Most previous epidemiological studies have been retrospective in design and therefore possibly complicated by selection bias. This bias may result in an emphasis on severe cases that require admission to hospital and may exclude mild cases as well as bleedings that are rapidly fatal. Because of methodological difficulties, only a few studies have overcome this problem by prospective, population based designs, and the study populations have usually been small. ${ }^{167111215172021}$ Concerning the completeness of case ascertainment in the present study, our data were collected at the various participating hospitals. It could, therefore, be argued that some patients with intracranial haemorrhage were not investigated at hospitals and consequently were excluded from our study. For instance, there may have been fatal cases in the community where necropsy was not performed, as well as elderly cases with minor neurological deficits who were not evaluated by CT. However, it has been estimated that only 3\%-5\% of stroke survivors in Sweden are treated out of hospital ${ }^{22}$ and we contacted the departments of pathology and forensic medicine to detect those who had died outside hospitals. Moreover, the CT rate of patients with stroke is high in Sweden. ${ }^{23}$ It seems likely, therefore, that very few patients with spontaneous intracranial haemorrhage have remained undetected in our study.

Spontaneous intracranial haemorrhage was in $96 \%$ due to either PICH or SAH (447 out of 466 cases) with overall annual incidence rates of 10.0 for SAH and 28.4 for PICH/100 000 when adjusted to the Swedish population. Of all diagnosed haemorrhages, only six cases $(1 \%)$ were shown to be caused by AVM and 13 cases (3\%) by brain tumour. The emphasis on this report is, therefore, made on the epidemiology of PICH and SAH. Primary intracerebral haemorrhage was, overall, almost three times as common as $\mathrm{SAH}$, which is in general agreement with data from an American population ${ }^{8}$ although their incidence rates were generally lower (six for SAH and 15 for intracerebral haemorrhage/ 100 000/year). However, as clearly demonstrated in the present study, differences in incidence rate between SAH and $\mathrm{PICH}$ depended to a large extent on age and sex. For example, women younger than 65 had the same risk of being affected by $\mathrm{SAH}$ and $\mathrm{PICH}$, whereas men older than 75 were up to 16 times more likely to have PICH. 
INCIDENCE OF SAH

Subarachnoid haemorrhage (aneurysmal and non-aneurysmal) was significantly more common in women (13.5/100 000/year) than in men (6.4/100 000/year), which has been reported by others. ${ }^{152425}$ The biological explanation for this sex difference is not known. ${ }^{32}$ The overall incidence rate of 10.0 for $\mathrm{SAH}$ found in the present study is in the range of several other studies (for review and metaanalysis, see Linn et $a l^{15}$ ) but contrasts with Finnish studies showing incidence rates of above $25 / 100000$ person-years. ${ }^{17}{ }^{24}$ Whether the remarkably high incidence rates in Finland have genetic causes or can be ascribed to environmental factors is unknown. It has been ruled out that the discrepancy would be attributed to differences in study design. ${ }^{15}$ An epidemiological study on the incidence of SAH in northern Sweden, being close to Finland and on the same latitude, would be of great interest in this regard.

There was a marked relation between age and incidence of SAH (fig 1 and table 1). Thus incidence rates increased dramatically in the 35-44 and 45-55 year age groups, after which they remained constant. This pattern is by contrast with the incidence rates of $\mathrm{PICH}$, which continued to grow with advancing age. Similar age dependent incidence curves for $\mathrm{SAH}$ with the main increases during early middle ages have been reported previously ${ }^{125}$ and probably reflect the time required for the vascular diseases to develop-that is, aneurym formation and growth. The reason for the obvious plateau in incidence rate in the middle and higher ages is unclear. It may be speculated that as aneurysms continue to develop at a relatively constant rate, they also rupture at the same rate in the middle and high age groups.

INCIDENCE OF PICH

We found incidence rates for PICH of 32 for women and 25 for men/100 000/year when adjusted to the Swedish population, which are in the upper range of previously published studies. Among modern studies where CT was used in the evaluation of patients with stroke, incidence rates ranged from 13-15/100 000/ year in Libya, France, and the United States ${ }^{6-8}$, 20-23 in England ${ }^{19}$ and up to 31-35 in Finland, Australia, and Italy. ${ }^{10-12}$ The reason for the high variability in incidence rates between studies or countries is not known and does not seem to be attributable to differences in study design. One possible explanation may be differences in age distribution as high age is strongly linked to increased risk of PICH. This interpretation is supported by the present incidence rates after adjustment for age to the world population, being only 13.9/100 000/ year. Although there may be real differences in incidence of PICH between populations, the use of CT in patients with acute stroke may vary among countries due to clinical tradition or availability. For instance, older patients with acute but minor neurological deficits may not always have CT but are instead regarded as having stroke of ischaemic or unknown origin, which would underestimate the true incidence of small haematomas. There may also be differences in the management of patients with very severe stroke who die at home or where the CT is omitted because of the clinical situation. The relative proportion of patients with stroke of uncertain origin is likely to vary between regions and hospitals, and is therefore difficult to estimate. ${ }^{12}$ It is noteworthy that as many as $65 \%$ of patients with PICH were alert or only slightly drowsy at admission to hospital in the present study.

There was a dramatic increase in incidence of PICH with advancing age from middle age onwards for both sexes. Thus, although the overall incidence rate/100 000/year was 28.4 , it was more than 160 for those older than 75 and on average only 2 for those below 45 years. The age dependent rise in incidence rate has been ascribed to increasing prevalence among elderly people of cerebral amyloid angiopathy ${ }^{26}$ and vasculopathy associated with chronic arterial hypertension. ${ }^{27}$ Interestingly, there was a drop in incidence for men in the $\geqslant 85$ compared with the 75-84 age group (208 $v 148$ per 100 000/year) which was not seen among women, but this trend was not statistically significant. A similar pattern has been seen in four other studies ${ }^{9-12}$ but not in others. ${ }^{6-8}$

\section{LOCATION OF HAEMATOMA}

More than half of the PICH were categorised as lobar, which is higher than previously reported. Earlier studies typically report a proportion of lobar haematomas of $32 \%-40 \%{ }^{9-11}{ }^{28}$ However, some of these studies define a subgroup of large haematomas as "massive supratentorial" $(9 \%)^{9}$ or "massive cortical/subcortical" $(22 \%)^{11}$ where the origin of the haematoma could not be determined. In the present study we ascribed these large haematomas to either the lobar (cortical/ subcortical) or central group. It is also possible that a high rate of CT investigations in patients with acute stroke, also in those with minor neurological deficits, which is the case in the present study, may result in a higher number of detected small lobar haematomas. In addition, as lobar haematomas were relatively more common in the highest age groups in the present study (see below), a high rate of CT investigations in an increasing elderly population (as the Swedish one) would yield a high proportion of lobar haematomas. The relative proportions of haematomas in the brainstem $(4 \%)$ and the cerebellum $(8 \%)$ are similar to those previously reported. ${ }^{9-1128}$

Although sex did not have any relation to haematoma location, advancing age increased the proportion of supratentorial haemorrhages (lobar and central) relative to those of infratentorial (cerebellar and brainstem) location. This was particularly evident for the lobar haematomas, which accounted for almost $60 \%$ of all PICH among those older than 75. This is in contradiction with a study by Broderick et $a l^{28}$ who concluded that the proportion of lobar haematomas does not increase with advancing age. An increasing proportion of lobar haematomas, as seen in the present study is, however, in accordance with the high presence of 
amyloid angiopathy in cortical and subcortical vessels in elderly people. ${ }^{26} 2930$

RISK FACTORS

Subarachnoid haemorrhage

Age and sex were significant risk factors for SAH. A history of hypertension was documented in $28 \%$ of cases, which is similar to the proportion seen in an American population of patients with SAH. ${ }^{31}$ Hypertension has, indeed, been suggested as a risk factor for SAH although the scientific evidence for this association is relatively limited. ${ }^{32}$ Interestingly, the prevalence of prior stroke $(7 \%)$ or heart disease (11\%) was lower than that seen among patients with PICH (19 and 28\%, respectively) and not a single patient with SAH was treated with anticoagulation compared with $12 \%$ for the patients with PICH. This further confirms the fact that SAH and PICH differ not only in clinical characteristics but also in the underlying pathophysiological mechanisms by which they occur. Other proposed risk factors for $\mathrm{SAH}$, such as smoking, oral contraceptives, and alcohol, ${ }^{32}$ were not analyzed in our study.

\section{Primary intracerebral haemorrhage}

There were no significant differences in the prevalence of the various risk factors for the different PICH locations. Thus, age and sex, as well as presence of hypertension, diabetes mellitus, prior stroke, heart disease, anticoagulation, and antiplatelet therapy were similarly distributed among the various haematoma sites. Similar findings have been made previously for hypertension ${ }^{1127}{ }^{28}$ and do not support the widespread term "hypertensive haemorrhage" traditionally linked to PICH in the deep nuclei, brain stem, or cerebellum. A history of hypertension was found in $37 \%$ of all cases with PICH in the present study, which is lower than the $55 \%-72 \%$ previously reported. ${ }^{9} 112728$ However, we did not search for undiagnosed cases of hypertension by analyzing electrocardiograms for left ventricular hypertrophy, chest radiographs for cardiomegaly, or blood pressures measured after the onset of stroke. It may be argued that the similar profiles of risk factors among the different haematoma locations would suggest that the underlying vascular diseases causing PICH are the same regardless of site. This assumption is, however, in contradiction with the above mentioned high prevalence of amyloid angiopathy in the cortical/ subcortical regions of elderly people and the high proportion of lobar haematomas in patients older than 75 .

The proportions of patients on antiplatelet therapy $(22 \%)$, anticoagulation $(12 \%)$, or with diabetes mellitus $(10 \%)$, prior stroke $(19 \%)$ or heart disease $(28 \%)$ is in general agreement with those described previously, ${ }^{10} 273334$ and reflect the generally high prevalence of arteriosclerotic vascular disease among patients with PICH. Whether the presence of arteriosclerosis in itself increases the risk of PICH or only coexists with other established risk factors (high age, hypertension, anticoagulation) is not clear. Smoking or aspirin intake do not seem to be independent risk factors for PICH. ${ }^{35}{ }^{36} \mathrm{It}$ should be pointed out, however, that the present study was not primarily designed to study the importance of risk factors. Only their prevalence among haemorrhage subtypes was studied here and any firm conclusions concerning their relative importance could, therefore, not be drawn.

In summary, we have obtained reliable data on the epidemiology of PICH and SAH by adopting a prospective design in a large population (1.14 million). We found a high overall incidence of PICH (28.4/100 000/year), which was almost three times as common as $\mathrm{SAH}$ (10.0/100 000/year), and there was a very strong influence of age and sex. A high proportion of lobar haematomas (overall $51.6 \%$ ) was seen, especially in the higher age groups. Hypertension was found in $37 \%$ per cent of patients with PICH with no significant difference between different haematoma locations. Oral anticoagulation treatment seemed to be an important risk factor for PICH but not for SAH. Our study further emphasises the importance of spontaneous intracranial haemorrhage as a relatively common medical catastrophy, particularly in societies with enlarging elderly populations.

We thank Roland Perfekt at the Southern Swedish Tumour Registry, Lund University Hospital for help with the statistical analysis. The study was supported by grants from the Neurosurgical Research Foundation at the University of Lund, the Rut and Erik Hardebo Donation Fund, the Foundation of the Rut and Erik Hardebo Donation Fund, the Foundation of
the Swedish Stroke Association and the Swedish Brain Foundation.

The following people and hospitals participated in the registration of patients with spontaneous intracranial haemorrhage: J Centergård and I Torstensson, Kristianstad Hospital; I Dahlqvist, Landskrona Hospital; H Karlsson, Karlskrona Hospital; C Lagerstedt and H Strömblad, Karlshamn Hospital, $S$ Lind-Pedersen, Ljungby Hospital; B Norrving, Lund University Hospital; L Svensson, Växjö Hospital; E Skarfors and J Kinnman, Halmstad Hospital; I Timberg, Hässleholm Hospital; P Träff, Ystad Hospital, J Wirén, Simrishamn Hospital, and B Ågren, Helsingborg Hospital.

1 Bamford J, Sandercock P, Dennis M, et al. A prospective study of acute cerebrovascular disease in the community: the Oxfordshire community stroke project 1981-6. 2. Incidence, case fatality rates and overall outcome at 1 year of cerebral infarction, primary intracerebral and subarachnoid haemorrhage. 7 Neurol Neurosurg Psychiatry 1990;53:1622.

2 Norrving B. Cerebral hemorrhage. In: Ginsberg $M$, Bogousslavsky J, eds. Cerebrovascular disease: pathophysiology, diagnosis, and managment. Oxford: Blackwell Science, 1999:1447-73.

3 Rowe CC, Donnan GA, Bladin PF. Intracerebral haemorrhage: incidence and use of computed tomography. BMF 1988;297:1177-8.

4 Drury I, Whisnant JP, Garraway WM. Primary intracerebral hemorrhage: impact of CT on incidence. Neurology 1984;34:653-7.

5 Hawkins GC, Bonita R, Broad JB, et al. Inadequacy of clinical scoring systems to differentiate stroke subtypes in
population-based studies. Stroke 1995;26:1338-42.

6 Ashok PP, Radhakrishnan K, Sridharan RA, et al. Incidence and pattern of cerebrovascular diseases in Benghazi, Libya. and pattern of cerebrovascular diseases in Beng

7 Giroud M, Gras P, Chadan N, et al. Cerebral haemorrhage Giroud $\mathrm{M}$, Gras $\mathrm{P}$, Chadan N, et al. Cerebral haemorrhage
in a French prospective population study. $\mathcal{F}$ Neurol Neurosurg Psychiatry 1991;54:595-8.

8 Broderick JP, Brott T, Tomsick T, et al. Intracerebral hemorrhage more than twice as common as subarachnoid hemorhage. F Neurosurg 1993;78:188-91.

9 Boonyakarnkul S, Dennis M, Sandercock P, et al. Primary intracerebral haemorrhage in the Oxfordshire community stroke project. 1. Incidence, clinical features and causes. Cerebrovasc Dis 1993;3:343-9.

10 Fogelholm R, Nuutila M, Vuorela AL. Primary intracerebral haemorrhage in the Jyväsklä region, central Finland, 1985-9: incidence, case fatality rate, and functional Anderson CS, Chakera TM, Stewart-Wynne E, et al. Spectrum of primary intracerebral haemorrhage in Perth, Western Australia, 1989-90: incidence and outcome. F Neurol Neurosurg Psychiatry 1994;57:936-40. 
12 Lauria G, Gentile M, Fassetta G, et al. Incidence and prognosis of stroke in the Belluno province, Italy. First-year 93 .

13 Broderick JP, Brott $\mathrm{T}$, Tomsick $\mathrm{T}$, et al. The risk of subarachnoid and intracerebral hemorrhages in blacks as compared with whites. N Engl F Med 1992;326:733-6.

14 Rinkel GJ, Djibuti M, van Gijn J. Prevalence and risk of rupture of intracranial aneurysms: a systematic review. Stroke 1998;29:251-6.

15 Linn FH, Rinkel GJ, Algra A, et al. Incidence of subarachnoid hemorrhage: role of region, year, and rate of 625-9.

16 Ljunggren B, Säveland H, Brandt L, et al. Early operation and overall outcome in aneurysmal subarachnoid hemorrhage. f Neurosurg 1985;62:547-51.

17 Sarti C, Tuomilehto J, Salomaa V, et al. Epidemiology of subarachnoid hemorrhage in Finland 1983-5. Stroke 1991; 22:848-53.

18 Waterhouse J, Correa P, Muir C, et al, eds. Cancer incidence in five continents. Lyon, France: International Agency for Research on Cancer, 1976:456.

19 Rothman KJ, Modern epidemiology. Boston: Little, Brown, 1986.

20 Ueda K, Hasuo Y, Kiyohara Y, et al. Intracerebral hemorrhage in a Japanese community, Hisayama: incidence, changing pattern during long-term follow-up, and related factors. Stroke 1988;19:48-52

21 Longstreth WJ, Nelson LM, Koepsell TD, et al. Clinical course of spontaneous subarachnoid hemorrhage: a population-based study in King County, Washington. Neurology 1993;43:712-8.

22 Norris JW. Stroke management around the world. Cerebrovasc Dis 1994;4:430-40.

23 Stegmayr B, Asplund K, Hulter-Åsberg K, et al. Stroke units in their natural habitat: can results of randomized trials be reproduced in routine clinical practice? Riks-Stroke reproduced in routine clinical practio
Collaboration. Stroke. 1999;30:709-14.
24 Sivenius J, Heinonen OP, Pyorala K, et al. The incidence of stroke in the Kuopio area of east Finland. Stroke 1985;16: stroke in

25 Truelsen T, Bonita R, Duncan J, et al. Changes in subarachnoid hemorrhage mortality, incidence, and case fatality in New Zealand between 1981-3 and 1991-3. Stroke 1998;29:2298-303.

26 Vinters HV. Cerebral amyloid angiopathy. A critical review. Stroke 1987;18:311-24.

27 Brott T, Thalinger K, Hertzberg V. Hypertension as a risk actor for spontaneous intracerebral hemorrhage. Stroke 1986;17:1078-83

28 Broderick J, Brott T, Tomsick T, et al. Lobar hemorrhage in the elderly. The undiminishing importance of hypertension. Stroke 1993;24:49-51.

29 Masuda J, Tanaka K, Ueda K, et al. Autopsy study of incidence and distribution of cerebral amyloid angiopathy in Hisayama, Japan. Stroke 1988;19:205-10.

30 Vonsattel JP, Myers RH, Hedley WE, et al. Cerebral amyloid angiopathy without and with cerebral hemorrhages: a comangiopathy without and with cerebral hemorrhages: a com-

31 Phillips LI, Whisnant JP, O'Fallon WM, et al. The unchanging pattern of subarachnoid hemorrhage in a community. Neurology 1980;30:1034-40.

32 Longstreth WJ, Koepsell TD, Yerby MS, et al. Risk factors for subarachnoid hemorrhage. Stroke 1985;16:377-85.

33 Hart RG, Boop BS, Anderson DC. Oral anticoagulants and intracranial hemorrhage. Facts and hypotheses. Stroke 1995;26:1471-7.

34 Kase CS, Robinson RK, Stein RW, et al. Anticoagulantrelated intracerebral hemorrhage. Neurology 1985;35:9438.

35 Thrift AG, McNeil JJ, Forbes A, et al. Risk of primary intracerebral haemorrhage associated with aspirin and non-steroidal anti-inflammatory drugs: case-control study. BMF 1999;318:759-64.

36 Thrift AG, McNeil JJ, Donnan GA. The risk of intracerebral haemorrhage with smoking. The Melbourne Risk Factor Study Group. Cerebrovasc Dis. 1999;9:34-9. 